\title{
Charge Transport in Semiconductors with Multiscale Conformational Dynamics
}

\author{
Alessandro Troisi, ${ }^{1}$ David L. Cheung, ${ }^{1}$ and Denis Andrienko ${ }^{2}$ \\ ${ }^{1}$ Department of Chemistry and Centre of Scientific Computing, University of Warwick, Coventry, CV4 7AL, United Kingdom \\ ${ }^{2}$ Max Planck Institute for Polymer Research, Ackermannweg 10, 55128 Mainz, Germany
}

(Received 4 December 2008; published 20 March 2009)

\begin{abstract}
In partially ordered organic semiconductors, the characteristic times of nuclear motion are comparable to those of charge carrier dynamics. It is impossible to describe charge transport using either static disorder models or temperature averaged electronic Hamiltonians. We build a model Hamiltonian which allows the study of charge transport whenever carrier and nuclear dynamics are not easily separable. Performing nanoseconds long molecular dynamics of a columnar mesophase of a discotic liquid crystal and evaluating electronic couplings, we identify realistic parameters of the Hamiltonian. All modes which are coupled to the electron dynamics can be described in the model Hamiltonian by a limited number of Langevin oscillators. This method can be applied to systems with both slow (nanoseconds) and fast (hundreds of femtoseconds) nuclear motions, i.e., with both dynamic and static disorder.
\end{abstract}

PACS numbers: 72.80.Le, 71.23.-k, 72.80.Ph, 73.63.Bd

Whenever there is strong coupling between electron dynamics and large amplitude nuclear motion, there are two treatable limits: if the nuclear dynamics is much slower than the dynamics of charge carriers, the latter can be described by a Hamiltonian with static disorder based on simple assumptions on the electronic density of states and on the hopping rates between localized states [1]. Indeed, transport in disordered systems has successfully been modeled by assuming a hopping mechanism for charge transport [2-4]. On the other hand, fast nuclear dynamics can be accounted for by averaging out fast degrees of freedom in the electronic Hamiltonian [5], as is done in band renormalizations [6].

Partially ordered (e.g., semicrystalline or liquid crystalline) organic semiconductors form an important class of systems where neither of these two limits is valid. These materials, with discotic liquid crystals and conjugated polymers being representative examples, are normally soft, and hence can be easily solvated, spin coated, or ink-jet printed, which renders them cost-effective substitutes of inorganic semiconductors [7]. In spite of their importance for applications, the description of the charge carrier dynamics in such materials is extremely difficult as their conformational dynamics may take place over the same time scale as the electron dynamics, making it impossible to separate nuclear and electronic degrees of freedoms through standard methods [8].

Usually these systems are described by a model Hamiltonian with interacting electronic and nuclear degrees of freedom constructed on the basis of a number of assumptions (i.e., top-down) and treatable with some approximate method $[9,10]$. This approach has several drawbacks. First, one has to predefine the type of transport, e.g., adiabatic versus nonadiabatic [11], or the density of states distribution [12], as it is done in the Gaussian disorder model [2], or specific type of dynamics in the system, e.g., harmonic oscillators [13]. These assumptions are all system-specific and cannot be always justified. In soft systems, for example, the dynamics of collective variables, which in our case are conjugated units, is no longer harmonic. In other words, it is not possible to build a model Hamiltonian on the basis of simple intuition.

To remedy this situation, we propose a bottom-up construction: we first fully characterize the classical part of Hamiltonian by analyzing molecular dynamics of the system performed with force-field based simulations. The transfer integrals between the molecules are evaluated using quantum chemical calculations and are then used to build the model Hamiltonian. Finally, we investigate how structural fluctuations at all time scales below $100 \mathrm{~ns}$ affect the charge carrier dynamics, compare our results with experiments as well as the static disorder limit.

As a test system, we study a typical discotic liquid crystal, a derivative of hexabenzocoronene $[\mathrm{HBC}$, see Fig. 1(a)]. HBC self-organizes into columnar structures with conjugated molecules stacked on top of each other. Overlap of the electronic $\pi$-systems of molecules in the columns enables charge transport along them, rendering this material as a one-dimensional semiconductor, with charge mobilities up to $1 \mathrm{~cm}^{2} \mathrm{~V}^{-1} \mathrm{~s}^{-1}$ [14]. HBC undergoes structural transition from a crystalline herringbone mesophase to columnar liquid crystalline mesophase at about $370 \mathrm{~K}$ and has rich molecular dynamics with several slow and fast modes present in the spectra [15].

The columnar mesophase was constructed by placing 10 HBC molecules in each column [see Fig. 1(b)] and positioning 16 columns on a hexagonal lattice. The parameters of the equilibration and production runs are given in Refs. [16,17]. Transfer integrals were calculated for every pair of neighboring molecules using geometry-optimized rigid copies of molecules and frozen orbital approximation [18]. Note that HBC has two degenerate HOMO orbitals labeled here $a$ and $b$ [see Fig. 1(c)]. The effect of the side chains can be neglected as their orbital energies are several 

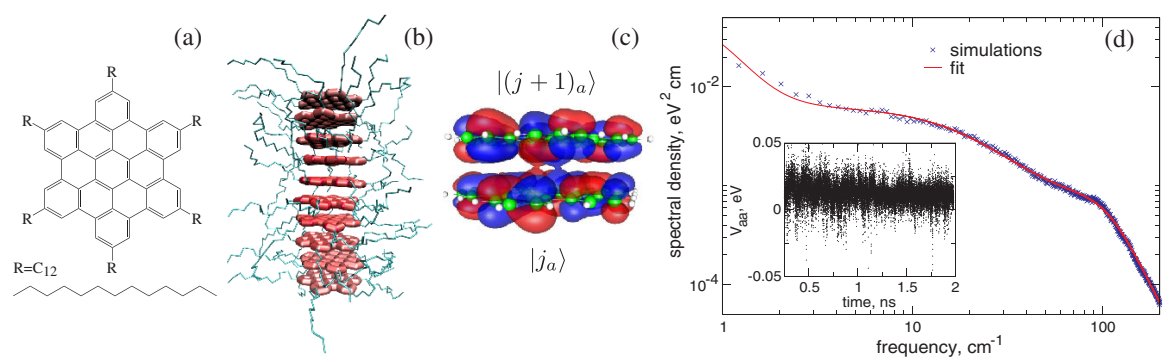

FIG. 1 (color online). (a) chemical structure (b) molecular dynamics snapshot (only one out of 16 columns shown) (c) representation of two HOMO orbitals on two consecutive sites (d) spectral density $Y_{a a}(\omega)$ resulting from the autocorrelation function of the intermolecular coupling $V_{a a}(t)$. Crosses are the simulation results, and the continuous line is the fitting used to parametrize the model Hamiltonian. The inset shows a portion of the $V_{a a}(t)$ function used in the calculation.

$\mathrm{eV}$ lower than the HOMO, and the poor interchain coupling makes them good insulators. The time dependent coupling $V_{a a}(t)$ between two neighboring $a$-HOMOs is depicted in the inset of Fig. 1(d). The spectral density $Y(\omega)$, defined as the Fourier transform of the autocorrelation function of the coupling $Y_{a a}(\omega)=\int\left\langle V_{a a}(0) V_{a a}(t)\right\rangle \times$ $\exp (-i \omega t) d t$, was computed for all the 4 couplings ( $a a$, $a b, b a, b b)$ between the degenerate HOMOs $a$ and $b$ in neighboring sites. The four spectral densities are found to be identical or differ by a constant $C$, i.e., $Y_{a a}(\omega)=$ $Y_{b b}(\omega)=C Y_{a b}(\omega)=C Y_{b a}(\omega)$, with $C=1.43$. The functions $Y$ collect the essential information on the nonlocal electron-phonon coupling resulting from hundreds of different nuclear modes per molecule. The function $Y_{a a}$, averaged among 6 different molecular pairs, is depicted in Fig. 1(d). The molecular motions that modulate the intermolecular hopping integrals span a continuous range from 0 to $200 \mathrm{~cm}^{-1}$ (corresponding to fluctuations in the $\sim 20$ ps time scale or slower). As the mobility of this material is of the order of $0.1-1 \mathrm{~cm}^{2} \mathrm{~V}^{-1} \mathrm{~s}^{-1}$, the residence time on one molecular unit is of the order of 5-50 ps (the distance between molecules is $3.6 \AA$ ). Obviously, one cannot assume here that the nuclear motions are much slower [19] or much faster [20] than the charge dynamics.

The analysis above points at a complex situation with hundreds of nuclear modes coupled to the electronic Hamiltonian and characteristic time scales that prevent any simple averaging or renormalization. It is possible, however, to build a semiclassical Hamiltonian which captures the essential physics but is still sufficiently simple for numerical treatment. We consider a one-dimensional system with two degenerate orbitals $\left|j_{a}\right\rangle$ and $\left|j_{b}\right\rangle$ per each molecular site. The electronic Hamiltonian $H^{\mathrm{el}}$ is a function of the nuclear modes $\left\{u_{j}^{(k)}\right\} . u_{j}^{(k)}$ indicates mode $k$ localized on molecule $j$. The modes modulate linearly the hopping integral between neighboring sites as

$$
\left\langle j_{a}\left|H^{\mathrm{el}}\right|(j+1)_{a}\right\rangle=\tau_{a a}+\sum_{k} \alpha_{a a}^{(k)}\left(u_{j+1}^{(k)}-u_{j}^{(k)}\right),
$$

where $\tau_{a a}$ is the average coupling, $\alpha_{a a}^{(k)}$ the Peierls (or nonlocal) electron-phonon coupling parameter, and analo- gous relations are valid also for the $a b, b a, b b$ couplings. In Ref. [13], a related model was used to describe the charge dynamics in an organic crystal assuming that the $\left\{u_{j}^{(k)}\right\}$ behave as simple harmonic oscillators (in the absence of charge). In the present case, the spectral density is a continuous function in the $0-200 \mathrm{~cm}^{-1}$ range, and we assume that the nuclear modes follow the dynamics of a Langevin oscillator:

$$
\begin{aligned}
m^{(k)} \ddot{u}_{j}^{(k)}= & -m^{(k)}\left(\omega^{(k)}\right)^{2} u_{j}^{(k)}-m^{(k)} \gamma^{(k)} \dot{u}_{j}^{(k)}+R^{(k)}(t) \\
& -\frac{\partial}{\partial u_{j}^{(k)}}\left\langle\psi(t)\left|H^{\mathrm{el}}\right| \psi(t)\right\rangle .
\end{aligned}
$$

The first three terms on the right-hand side are the forces acting on a conventional Langevin oscillator with mass $m^{(k)}$, frequency $\omega^{(k)}$, friction coefficient $\gamma$, and a Markovian random force $R^{k}(t)$, i.e., $\left\langle R^{(k)}\right\rangle=0$ and $\left\langle R^{(k)}(0) R^{(k)}(t)\right\rangle=2 m^{(k)} \gamma^{(k)} k_{B} T \delta(t)$. The last term represents the force on the oscillator $u_{j}^{(k)}$ due to the presence of the charge. The introduction of the random force allows one to describe a system with a complicated spectral density using only a limited number of oscillators. Alternatively, one can think that the many inactive modes of the system are reincorporated in the stochastic component of the Hamiltonian as a thermal bath. For the diagonal terms of the electronic Hamiltonian, we use

$$
\left\langle j_{a}\left|H^{\mathrm{el}}\right| j_{a}\right\rangle=\epsilon_{a}+\sum_{k} \lambda_{a}^{(k)} u_{j}^{(k)},
$$

where $\epsilon_{a}$ is the diagonal on-site energy (here set to zero) and $\lambda_{a}^{(k)}$ is the local (or Holstein) electron-phonon coupling parameter. Equation (2) can be integrated numerically $[21,22]$ by updating in two alternating steps the nuclear positions-velocities and the wave function.

The model described is a semiclassical version of the Holstein-Peierls model with the random force acting on the vibrational degrees of freedom. The crucial point is that, by adding this force, we can reproduce the spectral density of the molecular motion with a small number of oscillators, and therefore dramatically reduce the dimensionality of the 
problem. By fitting the computed spectral density [see Fig. 1(d)] by a linear combination of the spectral densities of Langevin oscillators [23], we verified that the realistic spectral density can be well reproduced by only three Langevin oscillators that modulate the intermolecular coupling between two close sites. The oscillator that dominates the spectral density has frequency $57.1 \mathrm{~cm}^{-1}$. A higher energy oscillator provides only a minor correction to the overall shape of the spectral density, while a very low frequency oscillator $\left(1.0 \mathrm{~cm}^{-1}\right)$ provides a quasi static disorder (in the time scale of the electron dynamics) and is very close to the lowest frequency that can be resolved with our computational method. The excellent quality of the fitting indicates that the extremely complicated time dependency of the electronic Hamiltonian can be captured by a numerically tractable model.

As the spectral densities for coupling $a a$ and $b b$ are identical, we have $\alpha_{a a}^{(k)}=\alpha_{b b}^{(k)}$. Moreover, as the spectral densities for coupling $a b$ and $b a$ differ only by the multiplicative constant $C$ from coupling $a a$ and $b b$, we have $\alpha_{a a}^{(k)}=\sqrt{C} \alpha_{a b}^{(k)}=\sqrt{C} \alpha_{b a}^{(k)}$. In principle, one should take into account the possible correlations between the fluctuation of the hopping integrals $a a, a b, b a$, and $b b$. We have, however, verified that the results do not change if we consider these integrals to be completely decoupled (i.e., 4 sets of distinct Langevin oscillators modulating independently the 4 couplings) or completely coupled (i.e., only one set of Langevin oscillators modulating all 4 couplings). For simplicity, we report here the results for completely decoupled oscillators.

We assumed that a single effective mode, $k=k_{H}$, distinct from the ones that modulate the intermolecular coupling, is responsible for the Holstein coupling. The standard values used for this mode $\left(\omega^{\left(k_{H}\right)}=1400 \mathrm{~cm}^{-1}\right.$, $\left.m^{\left(k_{H}\right)}=6 \mathrm{amu}\right)$, as discussed extensively elsewhere [22], derive from the known observation that the Holstein modes in conjugated molecules invariably involve $\mathrm{C}-\mathrm{C}$ stretching vibrations with typical vibrational energy around $1400 \mathrm{~cm}^{-1} \cdot \lambda_{a, b}=2.63 \mathrm{eV} / \AA$ were set to reproduce the computed reorganization energy of an isolated HBC molecule, $0.13 \mathrm{eV}$ [24], and $\gamma^{\left(k_{H}\right)}=0$.

The time evolution of the wave function and the nuclear coordinates are evaluated numerically [21] for $5 \mathrm{ps}$ with the time step of 0.0125 fs for a system with 300 molecules and periodic boundary conditions. The initial wave function $\psi(0)_{n}$ is taken to be one of the electronic Hamiltonian eigenfuctions at $t=0$, and the initial positions and velocities of the nuclear oscillators are taken from the Boltzmann distribution at the temperature of the simulation $(420 \mathrm{~K})$. Because of the dynamic disorder, the wave function is initially localized within a few molecular sites. Its spread is monitored by calculating $R_{n}^{2}(t) \equiv\left\langle\psi_{n}(t)\left|r^{2}\right| \psi_{n}(t)\right\rangle-\left\langle\psi_{n}(t)|r| \psi_{n}(t)\right\rangle^{2} \quad$ and then thermally averaged over 290 different initial wave functions to give $\left\langle R_{n}^{2}(t)\right\rangle$ and the diffusion coefficient $D=$ $\lim _{t \rightarrow \infty}\left\langle R_{n}^{2}(t)\right\rangle / 2 t$. The mobility is obtained from the Einstein relation $\mu=e D / k_{B} T$ [22].

The mobility computed with this method is $2.4 \mathrm{~cm}^{2} \mathrm{~V}^{-1} \mathrm{~s}^{-1}$, which is in reasonable agreement with the experimentally measured value of $0.1 \mathrm{~cm}^{2} \mathrm{~V}^{-1} \mathrm{~s}^{-1}$ [14], especially taking into account that we have no adjustable parameters. The discrepancy with experiment may be due to the presence of (practically) static defects. The dynamics of these defects is so slow that their motion is not coupled to charge dynamics, and one can treat them as static disorder. To estimate this contribution, we compare our result to the static limit, assuming that the charge carrier dynamics is much faster than the dynamics of nuclei.

To do this, kinetic Monte Carlo (KMC) time-of-flight simulations were performed on columns constructed from molecular dynamics snapshots [25]. Analyzing photocurrent transients, we obtained transient time $t_{\mathrm{Tr}}=$ $1.5 \times 10^{-9} \mathrm{~s}$, which results in mobility $0.67 \mathrm{~cm}^{2} \mathrm{~V}^{-1} \mathrm{~s}^{-1}$, in between experimentally measured and predicted from the model Hamiltonian. The reasons for the overestimation of the experimental mobility (energetic disorder due to polarization and electrostatic contributions, external contribution to the reorganization energy, etc.) are discussed in detail in Ref. [4]. The discrepancy between two theoretical predictions is then a measure of the mobility reduction due to long-range disorder, which leads to broadening of the distribution of hopping rates on a large scale, decreasing the mobility [2]. This effect is neglected in our model Hamiltonian, since the dynamics of the system is mapped onto the dynamics of two nearest neighbors, but can, in principle, be incorporated by adding an additional (slow) Langevin oscillator with initial conditions reflecting the distribution of transfer integrals.

As evident from the discussion above, the traditional distinction between charge transport models for ordered and for disordered materials is not useful in the case of mesophases. The thermally averaged electronic Hamiltonian in these systems is that of an ordered phase while the instantaneous electronic Hamiltonian is characteristic of a fully disordered system. Since the time scale of the fluctuations coincides with the time scale of the dynamics of interest, the problem can be related to analogous problems encountered in condensed phase chemical dynamics, e.g., electron transfer reactions controlled by an underlying nuclear dynamics $[26,27]$. The diffusion of ions in polymeric matrices is another example of an independent dynamics (the motion of the polymer) which affects the dynamic of interest (the motion of the ions) [28]. Interestingly, the formulation of the problem in terms of stochastic equations of motion is found to be very useful in our case as in the examples above.

In conclusion, we propose a two-stage approach to model the charge transport in semiconducting soft condensed matter systems. First, we evaluate the time depen- 
dent electronic Hamiltonian using a combination of molecular dynamics and quantum chemical methods. Second, we distil the complicated dynamics emerging from these computations into a semiclassical model Hamiltonian that retains the essential characteristics (correlation functions) of the real system. The use of a small set of Langevin oscillators allows an efficient representation of the system using only a limited number of degrees of freedom, and the mobility computed without adjustable parameters is in good agreement with the experimental one. Using this approach, the mobility of different discotics, e.g., perylenediimide [29] can be calculated from first principles, opening up an opportunity to formulate structure-mobility relations and aid rational design of these compounds. The proposed approach is generally applicable whenever it is not possible to separate conformational and electronic dynamics in materials with a high degree of order.

This work was partially supported by DFG via IRTG program between Germany and Korea and DFG Grant No. AN 680/1-1. A. T. and D. L.C. acknowledge the support of EPSRC. D. L. C. acknowledges conversations with Jochen Voss. Kurt Kremer is acknowledged for critical reading of the manuscript.

[1] P. A. Lee and T. V. Ramakrishnan, Rev. Mod. Phys. 57, 287 (1985).

[2] P. M. Borsenberger, L. Pautmeier, and H. Bassler, J. Chem. Phys. 94, 5447 (1991).

[3] R. Coehoorn, W. F. Pasveer, P. A. Bobbert, and M. A. J. Michels, Phys. Rev. B 72, 155206 (2005).

[4] J. Kirkpatrick, V. Marcon, K. Kremer, J. Nelson, and D. Andrienko, J. Chem. Phys. 129, 094506 (2008).

[5] J. D. Picon, M. N. Bussac, and L. Zuppiroli, Phys. Rev. B 75, 235106 (2007).

[6] T. Holstein, Ann. Phys. (N.Y.) 281, 706 (2000).

[7] F. J. M. Hoeben, P. Jonkheijm, E. W. Meijer, and A. P. H. J. Schenning, Chem. Rev. 105, 1491 (2005).

[8] D. L. Cheung and A. Troisi, Phys. Chem. Chem. Phys. 10, 5941 (2008).

[9] M. Hultell and S. Stafstrom, Phys. Rev. B 75, 104304 (2007).

[10] F. C. Grozema and L. D. A. Siebbeles, Int. Rev. Phys. Chem. 27, 87 (2008)

[11] R. A. Marcus, Rev. Mod. Phys. 65, 599 (1993).

[12] Charge Transport in Disordered Solids, edited by S. Branowski (Wiley, Chichester, 2006).

[13] A. Troisi and G. Orlandi, Phys. Rev. Lett. 96, 086601 (2006).

[14] A. M. van de Craats et al., Adv. Mater. 11, 1469 (1999).

[15] M. M. Elmahdy, G. Floudas, M. Mondeshki, H. W. Spiess, X. Dou, and K. Müllen, Phys. Rev. Lett. 100, 107801 (2008).
[16] D. Andrienko, V. Marcon, and K. Kremer, J. Chem. Phys. 125, 124902 (2006).

[17] V. Marcon, T. Vehoff, J. Kirkpatrick, C. Jeong, D. Y. Yoon, K. Kremer, and D. Andrienko, J. Chem. Phys. 129, 094505 (2008).

[18] J. Kirkpatrick, Int. J. Quantum Chem. 108, 51 (2008).

[19] J. J. Kwiatkowski, J. Nelson, H. Li, J.L. Bredas, W. Wenzel, and C. Lennartz, Phys. Chem. Chem. Phys. 10, 1852 (2008).

[20] M. A. Palenberg, R. J. Silbey, M. Malagoli, and J. L. Bredas, J. Chem. Phys. 112, 1541 (2000).

[21] H. Ness and A. J. Fisher, Phys. Rev. Lett. 83, 452 (1999).

[22] A. Troisi, Adv. Mater. 19, 2000 (2007).

[23] The spectral density of a time dependent variable $V(t)$ which is proportional to the displacement of a Langevin oscillator can be written as $\frac{1}{\pi} V^{2}\left[\gamma \omega_{0}^{2} /\left(\omega_{0}^{2}-\omega^{2}\right)^{2}+\right.$ $\left.\gamma^{2} \omega^{2}\right]$, with $\gamma$ and $\omega_{0}$ being the friction coefficient and the harmonic frequency. The parameters for the three oscillators obtained from fitting are $\left\langle\left(V^{(1)}\right)^{2}\right\rangle=0.108 \mathrm{eV}^{2}$, $\omega_{0}^{(1)}=1.0 \mathrm{~cm}^{-1}, \gamma^{(1)}=1.66 \mathrm{~cm}^{-1},\left\langle\left(V^{(2)}\right)^{2}\right\rangle=0.301 \mathrm{eV}^{2}$, $\omega_{0}^{(2)}=57.1 \mathrm{~cm}^{-1}, \gamma^{(2)}=196 \mathrm{~cm}^{-1},\left\langle\left(V^{(3)}\right)^{2}\right\rangle=0.144 \mathrm{eV}^{2}$, $\omega_{0}^{(3)}=106 \mathrm{~cm}^{-1}, \gamma^{(3)}=116 \mathrm{~cm}^{-1}$. The electron-phonon coupling terms, $\alpha^{(k)}$ appearing in Eq. (1), are related to the fitting parameters as $\alpha_{a a}^{(k)}=\sqrt{\left\langle\left(V^{(k)}\right)^{2}\right\rangle m^{(k)}\left(\omega^{(k)}\right)^{2} / 2 k_{B} T}$. $m^{(k)}=600 \mathrm{amu}$ (the HBC core mass) for $k=1,2,3$.

[24] J. Kirkpatrick, V. Marcon, J. Nelson, K. Kremer, and D. Andrienko, Phys. Rev. Lett. 98, 227402 (2007).

[25] For KMC simulations, columns of molecules produced by MD are stacked periodically to produce a stack $L=1 \mu \mathrm{m}$ thick. A uniform field is applied along the stack, a charge carrier representing either an electron or a hole is introduced near one end, and the charge drift is simulated by a continuous-time random walk algorithm. Simulated transients are averaged over different starting positions of the charge and 200 MD snapshots. This method is analogous to the experimental situation where charges are generated in parallel columns, and the measured transient is the sum of displacement currents from all columns. Note that there is no a priori average of the electronic density of states as in other methods. The charge mobility is obtained from the transient time $t_{\mathrm{Tr}}$ of the simulated transient via $L=F t_{\mathrm{Tr}}$, where $L$ is the stack thickness. $t_{\operatorname{Tr}}$ is taken as the point of intersection of the two asymptotes to the simulated photocurrent plotted on a log-log plot, as would be done in a time-of-flight experiment. We used external field $F=$ $10^{5} \mathrm{~V} \mathrm{~cm}^{-1}$. A detailed description of the mapping of the snapshots on to a lattice and $\mathrm{KMC}$ simulations is given in Refs. [4,24].

[26] Y. A. Berlin, F. C. Grozema, L. D. A. Siebbeles, and M. A. Ratner, J. Phys. Chem. C 112, 10988 (2008).

[27] I. A. Balabin, D. N. Beratan, and S. S. Skourtis, Phys. Rev. Lett. 101, 158102 (2008).

[28] A. Nitzan and M. A. Ratner, J. Phys. Chem. 98, 1765 (1994).

[29] V. Marcon, J. Kirkpatrick, W. Pisula, and D. Andrienko, Phys. Status Solidi (b) 245, 820 (2008). 